RESEARCH ARTICLE

\title{
Optical control of transverse motion of ionization injected electrons in a laser plasma accelerator
}

\author{
Jie Feng ${ }^{1,2, \dagger}$, Yifei $\mathrm{Li}^{3, \dagger}$, Jinguang Wang ${ }^{3}$, Dazhang $\mathrm{Li}^{4}$, Changqing $\mathrm{Zhu}^{3}$, Junhao $\mathrm{Tan}^{3}$, Xiaotao Geng ${ }^{3}$, \\ Feng Liu ${ }^{1,2}$, and Liming Chen ${ }^{1,2}$ \\ ${ }^{1}$ Key Laboratory for Laser Plasmas (MoE), School of Physics and Astronomy, Shanghai Jiao Tong University, Shanghai 200240, \\ China \\ ${ }^{2}$ IFSA Collaborative Innovation Center and School of Physics and Astronomy, Shanghai Jiao Tong University, Shanghai 200240, \\ China \\ ${ }^{3}$ Beijing National Research Center of Condensed Matter Physics, Institute of Physics, CAS, Beijing 100190, China \\ ${ }^{4}$ Institute of High Energy Physics, CAS, Beijing 100049, China \\ (Received 28 June 2020; revised 7 December 2020; accepted 10 December 2020)
}

\begin{abstract}
We demonstrate an all-optical method for controlling the transverse motion of an ionization injected electron beam in a laser plasma accelerator by using the transversely asymmetrical plasma wakefield. The laser focus shape can control the distribution of a transversal wakefield. When the laser focus shape is changed from circular to slanted elliptical in the experiment, the electron beam profiles change from an ellipse to three typical shapes. The three-dimensional particlein-cell simulation result agrees well with the experiment, and it shows that the trajectories of these accelerated electrons change from undulating to helical. Such an all-optical method could be useful for convenient control of the transverse motion of an electron beam, which results in synchrotron radiation from orbit angular momentum.
\end{abstract}

Keywords: electron beam; laser plasma; transverse motion; wakefield acceleration

\section{Introduction}

The concept of laser plasma wakefield accelerators (LWFAs) was first proposed by Tajima and Dawson ${ }^{[1]}$. Over the past few decades, LWFAs have become increasingly mature and have recently exhibited stable ${ }^{[2]}$, low divergence (milliradians) ${ }^{[3]}$ and energy tunable ${ }^{[4]}$ electron bunches with a charge at the picocoulomb level ${ }^{[5]}$. An electron beam is most efficiently produced in the 'bubble' regime ${ }^{[6]}$, which requires laser pulses that are both intense (normalized vector potential $a_{0}>1$ ) and short (pulse duration $\tau \leq 2 \pi c / \omega_{\mathrm{p}}$, where $\omega_{\mathrm{p}}$ is the plasma frequency). The ponderomotive force of these laser pulses propagating in an underdense plasma pushes the background electrons away from the high-intensity regions and drives a relativistic plasma wave. The wave consists of a string of ion cavities (also referred to as 'bubbles'),

Correspondence to: D. Li, Institute of High Energy Physics, CAS, Beijing 100049, China; L. Chen, School of Physics and Astronomy, Shanghai Jiao Tong University, Shanghai 200240, China. Email: lidz@iphy.ac.cn (D. Li); Email: 1mchen@sjtu.edu.cn (L. Chen)

${ }^{\dagger}$ These authors contributed equally to this work. and the electrons trapped inside can be accelerated by the electrostatic field set up by the separation of electrons and ions. Moreover, these accelerated electrons will also oscillate in the plasma wakefield with betatron frequency $\omega_{\beta}=$ $\omega_{\mathrm{p}} / \sqrt{2 \gamma}$ and emit synchrotron radiation ${ }^{[7]}$. There are several methods of electron capture, including ponderomotive force injection ${ }^{[8]}$, colliding laser pulse injection ${ }^{[9]}$, plasma density gradient injection ${ }^{[10]}$ and transverse self-injection ${ }^{[11-13]}$. With these methods, the injected direction of electrons is hard to control, and these injection processes are not easy to achieve in experiment. In contrast, another method is ionization-induced injection ${ }^{[14-16]}$, which is used in this study. Owing to the different ionization potential levels of high $Z$ atoms ${ }^{[15,17-19]}$ (such as nitrogen), the outer shell electrons can be ionized instantaneously by the rising edge of the laser pulses ( $98 \mathrm{eV}$ for $\mathrm{N}^{+5}$ requires an intensity of $\sim 2 \times 10^{16} \mathrm{~W} / \mathrm{cm}^{2}$ ) and pushed away. The inner shell electrons $\left(552 \mathrm{eV}\right.$ for $\mathrm{N}^{+6}$ requires an intensity of $\left.\sim 1 \times 10^{19} \mathrm{~W} / \mathrm{cm}^{2}\right)$ are ionized close to the peak of the laser intensity envelope. These ionized electrons will appear at rest and slip backward relative to the laser pulses and the wake. The electrons are trapped after gaining enough energy from the

(C) The Author(s), 2021. Published by Cambridge University Press in association with Chinese Laser Press. This is an Open Access article, distributed under the terms of the Creative Commons Attribution licence (http://creativecommons.org/licenses/by/4.0/), which permits unrestricted re-use, distribution, and reproduction in any medium, provided the original work is properly cited. 
longitudinal electric field of the first period of the wake to move at the phase velocity of the wake and will gain additional energy ${ }^{[15]}$. Ionization injection is a more controllable method, regarded particularly for its stability ${ }^{[14,20,21]}$. Moreover, these trapped electrons mainly oscillate along the direction of laser polarization in the ion cavity.

Owing to the fact that the plasma wakefield has a transverse electric field of tens of gigavolts per metre and the radius of a plasma bubble is limited to several micrometres $^{[22]}$, it is difficult to find a strong enough external electric field or magnetic field for controlling the transverse motion of an electron beam in a bubble, especially helical motion. Moreover, Luo et al. ${ }^{[23]}$ simulated and acquired the helical motion of an electron beam and elliptically polarized radiation by laser pulses incident at a skew angle to the axis of the plasma waveguide, but this method was hard to achieve in experiment. Thaury et al. ${ }^{[24]}$ used one laser pulse to drive an asymmetrical plasma wakefield and another pulse of colliding injection to achieve the helical motion of an electron beam, but the process of colliding injection is not easy to control and has low repetition probability. In addition, Chang et al. ${ }^{[25]}$ also generated the helical motion of an electron beam and circularly polarized radiation by using a petawatt-class circularly polarized laser pulse interaction with near-critical density plasma, but the divergences of the electron beam and radiation were very large.

In this paper, we propose a simple all-optical method to control the transverse motion of the ionization injected electron beam by changing the evolution of the plasma wakefield transversal distribution. We also use three-dimensional particle-in-cell (3D-PIC) simulation to verify our experimental results and analyze the dynamics of electron transverse motion.

\section{Experimental setup and results}

The experiment was performed at the Key Laboratory for Laser Plasmas at Shanghai Jiao Tong University using the 100 TW laser system: a Ti:sapphire laser operating at $10 \mathrm{~Hz}$ repetition rate with a central wavelength $\lambda_{0}$ of $795 \mathrm{~nm}$. In the experiment, the system delivered $3 \mathrm{~J}$ p-polarized pulses with duration of $30 \mathrm{fs}$ in FWHM. The experimental setup is shown in Figure 1. The laser pulse was focused by an $f / 20$ off-axis parabolic (OAP) mirror to a vacuum spot size close to a Gaussian intensity distribution with FWHM diameter of $30 \mu \mathrm{m}$ and containing $30 \%$ energy. By adjusting the OAP mirror posture to the optimal position, the intensity distributions and spot shapes on, in front of and behind the focal plane were all approximately circular, as shown in Figure 1(a). The vacuum-focused laser intensity can reach up to $6.5 \times 10^{18} \mathrm{~W} / \mathrm{cm}^{2}$, for which the corresponding normalized vector potential $a_{0}$ is $\sim 1.7$. The plasma target was formed using a $1.2 \mathrm{~mm} \times 4 \mathrm{~mm}$ supersonic gas jet, which can generate well-defined uniform gas density profiles in the range of $1 \times 10^{17} \mathrm{~cm}^{-3}$ to $3 \times$ $10^{19} \mathrm{~cm}^{-3}$, as the gas stagnation pressure is changed ${ }^{[26-28]}$. The laser focal plane was located at the front edge of the gas jet. The experimental results were obtained using pure nitrogen for ionization-induced injection, and the gas stagnation pressure was set at 1.2 bar $\left(1\right.$ bar $\left.=10^{5} \mathrm{~Pa}\right)$, corresponding to the background plasma density of $6 \times$ $10^{18} \mathrm{~cm}^{-3}$, considering the outer shell electrons of nitrogen were fully ionized. A top-view ${ }^{[29]}$ system, consisting of a 14-bit charge-coupled device (CCD) and a low-pass filter, was used to monitor the interaction position and the length of time-integrated plasma channel, as shown in Figure 1(c). The electron beams emitted from the plasma channel were

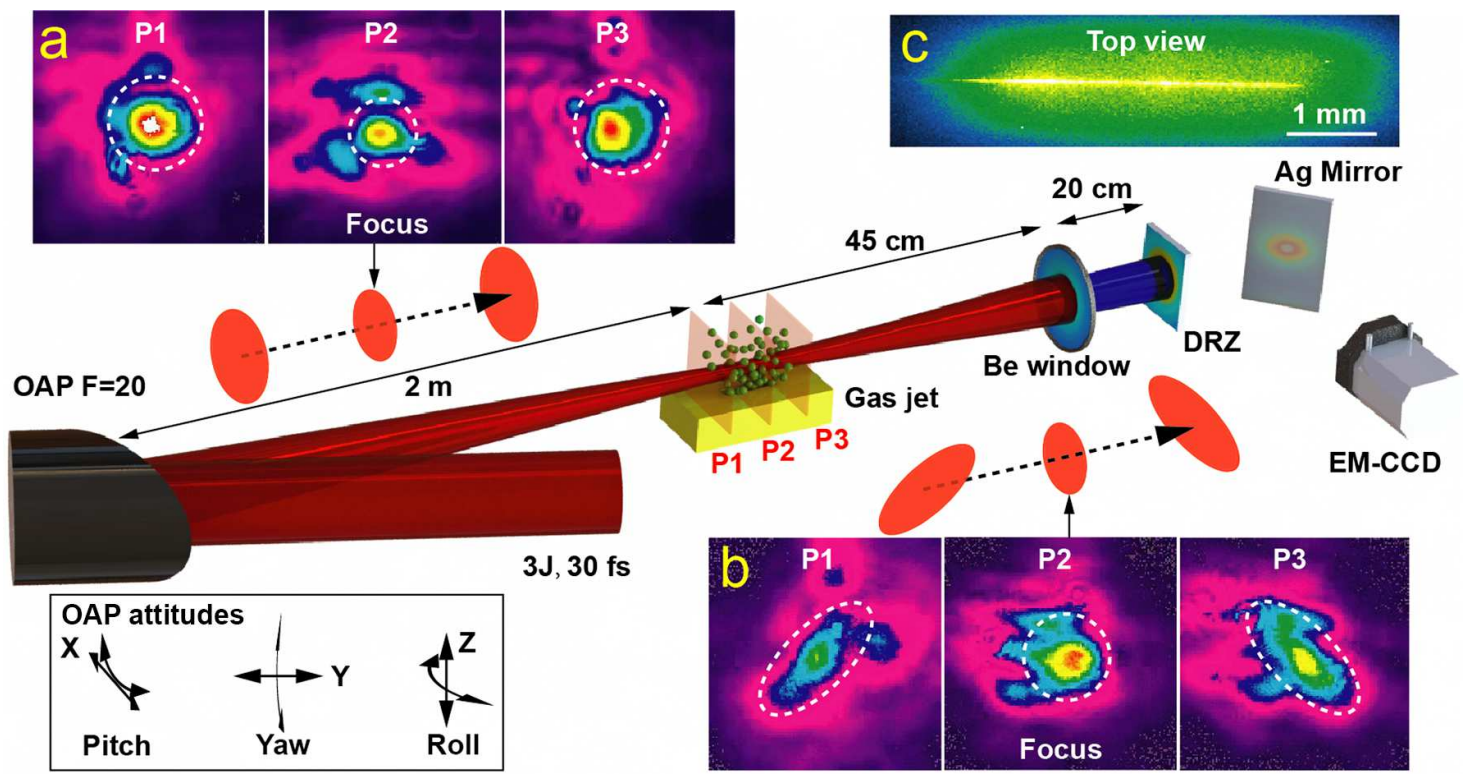

Figure 1. Experimental setup. (a) Laser intensity distribution measured in front of, at and behind, respectively, the focal spot in the case of a perfect focus situation. (b) Laser intensity distribution measured after adjusting the posture of the OAP mirror. (c) The top-view image of the plasma channel. 
detected by a DRZ fluorescent screen coupled with a 16bit electron-multiplying CCD (EM-CCD). The laser pulses were blocked by a beryllium window with a thickness of $350 \mu \mathrm{m}$. However, in order to control the transverse motion of the electron beam by using an asymmetrical transverse wakefield, we adjusted the OAP mirror postures of yaw, pitch and roll to generate the laser intensity distribution of a slanted $45^{\circ}$ ellipse and a $135^{\circ}$ ellipse in front of and behind the focal spot, respectively, as shown in Figure 1(b). Here, $45^{\circ}$ and $135^{\circ}$ refer to the included angle between the elliptical long axis and the horizontal axis.

The experimental results of the electron beam spots driven by laser pulses for the two kinds of focus shape are shown in Figure 2. When the posture of the OAP mirror is in the optimal position, the typical shape of these electron beam spots is an ellipse with its long axis in the horizontal direction ( $y$-direction), as shown in the fourth column $\left(0^{\circ}\right)$. As in Figure 1(b), the experimental results are shown in the first three columns of Figure 2, which demonstrate three different types of ellipse with the long axis in the directions of $45^{\circ}$, $90^{\circ}$ and $135^{\circ}$, respectively. To explain the results and analyze the dynamics of the electron beam in the plasma wakefield, we also carried out 3D-PIC simulations, as described in the following sections.

To acquire the energy spectrum and charge of the electron beam, a rectangle dipole magnet and an imaging plate (Fujifilm IP-SR series) were used. The magnet, of $8 \mathrm{~cm} \times$ $8 \mathrm{~cm}$ with a magnetic field intensity of $0.98 \mathrm{~T}$, was placed $50 \mathrm{~cm}$ away from the gas target to deflect the electron beam. The imaging plate was placed $7 \mathrm{~cm}$ from the exit of the magnet to record the deflected electron beam, and the imaging plate was calibrated for charge calculation ${ }^{[30]}$. The deflected electron distributions and calculated charges for five consecutive shots are shown in Figure 3. The electron beam in the case of symmetrical focus has higher maximum electron energy and charge ( 360 $\pm 8 \mathrm{MeV}, \sim 129 \pm 12 \mathrm{pC})$ than in the case of asymmetrical focus $(\sim 327 \pm 23 \mathrm{MeV}$, $\sim 91 \pm 13 \mathrm{pC}$ ). In this experiment, although the laser pulses with asymmetrical focus can drive the process of the LWFA, the electron beam parameters and stability have been sacrificed to a certain extent.

\section{3D-PIC simulation and results}

The 3D-PIC simulations were carried out using KLAPS code $^{[31,32]}$, and the tunnel-ionization model was adopted for field ionization. The simulation box size was $50 \mu \mathrm{m} \times$ $60 \mu \mathrm{m} \times 60 \mu \mathrm{m}$ with $1500 \times 450 \times 450$ cells in the $x$-, $y$ - and $z$-directions, respectively, and one cell contained two macro particles. In addition, a third-order time interpolation for the magnetic field was used in the simulation. P-polarized (ydirection) $800 \mathrm{~nm}$ laser pulses with $a_{0}=1.7$ were focused to a radius of $15 \mu \mathrm{m}$ at $x=50 \mu \mathrm{m}$ behind the front edge of the nitrogen gas. The pulse had a Gaussian transverse profile and sine-squared longitudinal shape with pulse duration of $30 \mathrm{fs}$ (FWHM). The neutral nitrogen longitudinal profile had a 100 $\mu \mathrm{m}$ up-ramp followed by a $2 \mathrm{~mm}$ long plateau with uniform density of $6 \times 10^{17} \mathrm{~cm}^{-3}$.

If a laser pulse with the focus situation shown in Figure (b) is propagating and self-focusing in the plasma, the shape of the laser spot will change from a slanted $45^{\circ}$ ellipse to a circle and then to a slanted $135^{\circ}$ ellipse. The process of laser spot shape change will continue until the laser pulses cannot sustain self-focusing in the plasma. Therefore, in order to

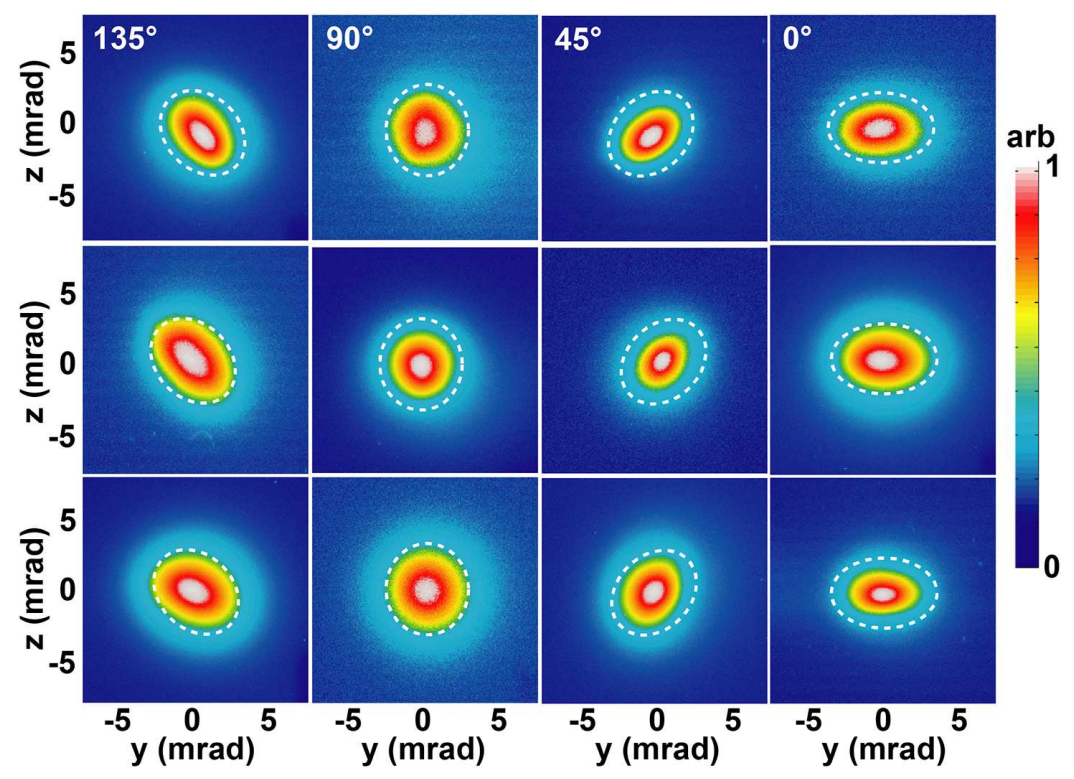

Figure 2. Electron beam spatial distribution. The first three columns are driven by the asymmetrical focus, showing three different typical shapes. All of these electron beam profiles were acquired under the same experimental conditions. The last column is driven by the symmetrical focus. All of the angles refer to the included angle between the elliptical long axis and the horizontal axis. 

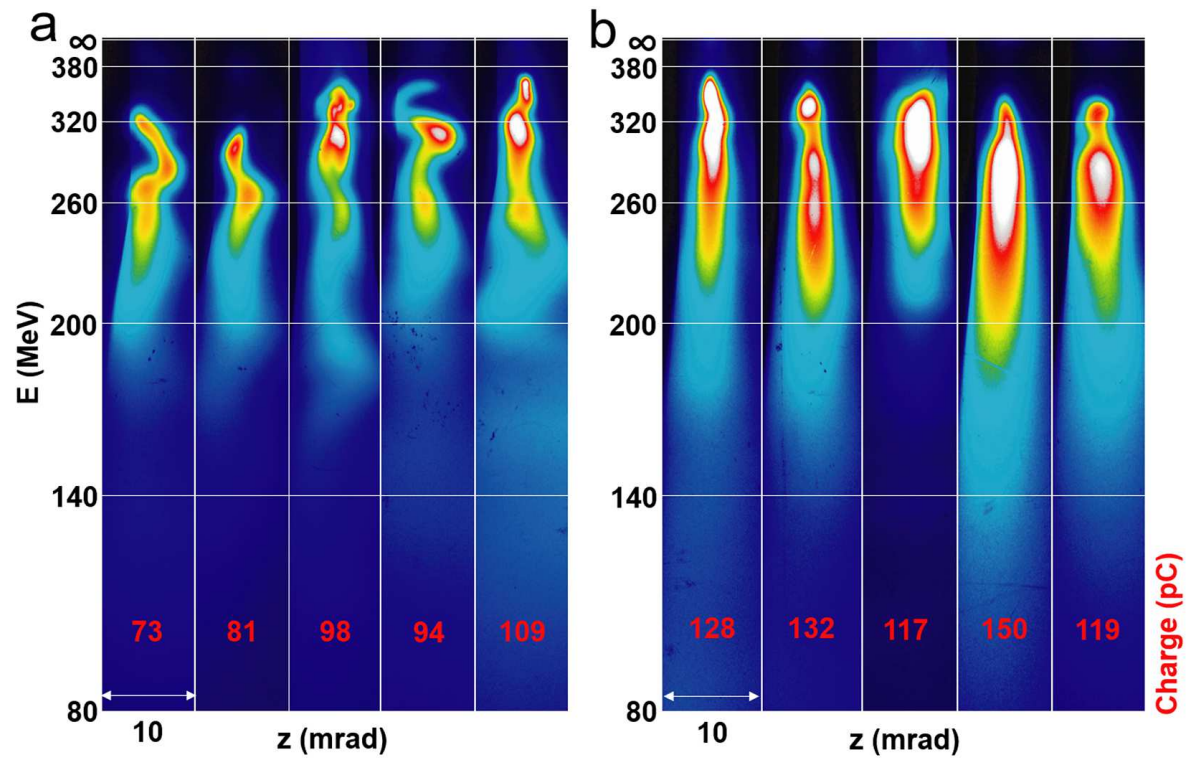

Figure 3. Deflected electron distributions and charges for five consecutive shots. The red numbers are the total charges for electron energy above $80 \mathrm{MeV}$. (a) Asymmetrical focus. (b) Symmetrical focus.
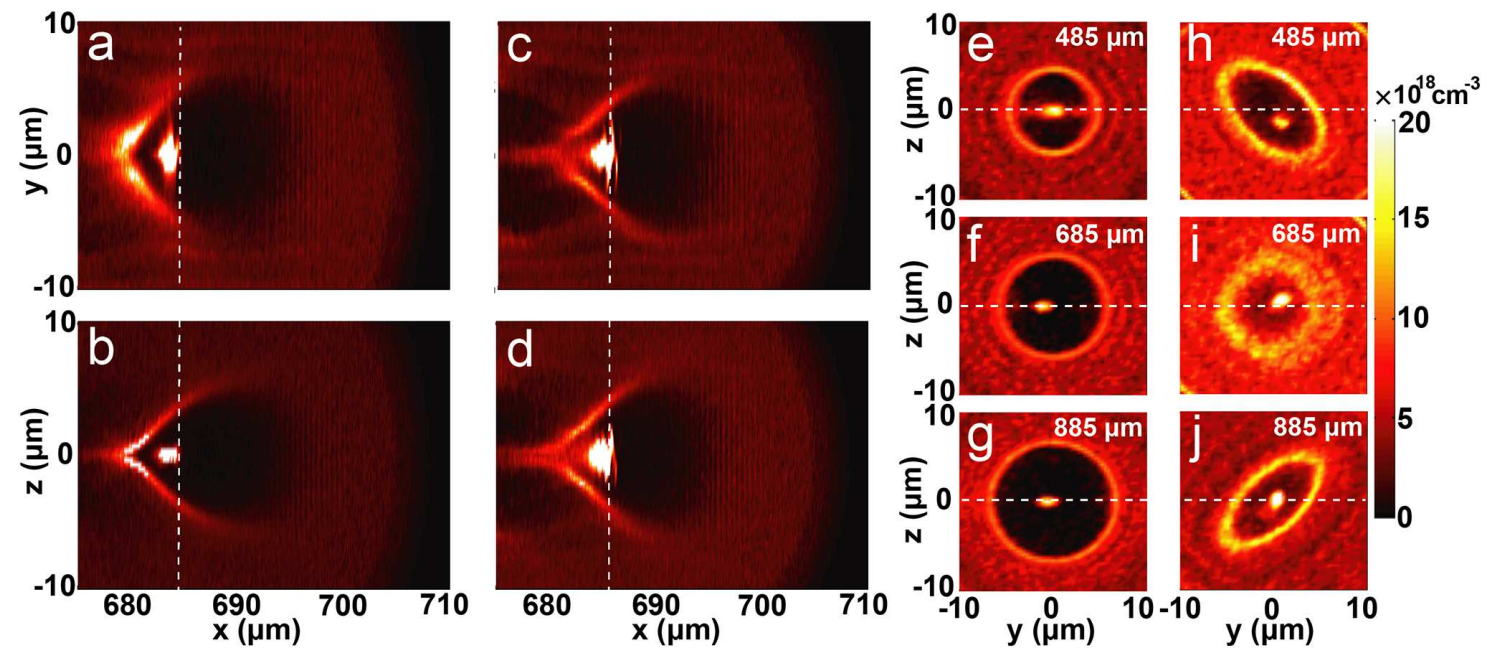

Figure 4. Laser plasma wakefield acceleration in 3D-PIC simulations. (a), (b) The cross-sections of the plasma bubble in the $X Y$ and $X Z$ planes, respectively, driven by the symmetrical laser spot. (c), (d) The cross-sections driven by the asymmetrical laser spot. (e)-(g) The cross-sections (in the $Y Z$ plane) of the plasma bubble at different propagation positions, corresponding to the case of the symmetrical spot. (h)-(j) The cross-sections corresponding to the case of the asymmetrical focal spot at different propagation positions.

study the process of this laser pulse propagation and selffocusing in the plasma, and the influence of the asymmetrical laser focus on the plasma wakefield acceleration, the asymmetrical laser intensity distribution in front of the focal plane was set according to the intensity distribution measured in the experiments (as shown in Figure 1(b)), and the electricfield intensity distribution is expressed as

$$
\begin{array}{r}
E(x, y, z)=E_{0} \cdot \sqrt{w_{0} / \mathrm{rs}(x)} \cdot \exp \left\{-\left[\frac{(y \cdot \cos \theta-z \cdot \sin \theta)^{2}}{2}\right.\right. \\
\left.\left.+\frac{(y \cdot \cos \theta+z \cdot \sin \theta)^{2}}{0.5}\right] / \mathrm{rs}(x)^{2}\right\},
\end{array}
$$

where $\operatorname{rs}(x)=w_{0} \cdot \sqrt{1-\left(x-x_{0}\right)^{2} / z_{\mathrm{R}}^{2}}, x_{0}$ is the longitudinal position of the focal plane and $z_{R}$ is the Rayleigh length. $\theta=45^{\circ}$ is the rotation angle (clockwise direction) of the long axis of the ellipse shape.

The simulation results are shown in Figure 4. In the case of the symmetrical laser spot, the electron beam in the plasma bubble has a bigger transversal size (FWHM) in the $y$-direction $(\sim 3 \mu \mathrm{m})$ than in the $z$-direction $(\sim 0.5 \mu \mathrm{m})$, owing to the ionization injection, as shown in Figures 4(a) and 4(b). The simulation results also demonstrate the crosssections ( $Y Z$ plane) of the bubble, and that the electron beam oscillates mainly in the $X Y$ plane, as shown in Figures 4(e) and 4(f). However, in the case of the asymmetrical 
laser spot, the transversal size of the electron beam in the $y$-direction $(\sim 2 \mu \mathrm{m})$ is reduced but increasing noticeably in the $z$-direction $(\sim 2 \mu \mathrm{m})$, as shown in Figures 4(c) and 4(d). Moreover, the shape evolution of the cross-section is similar to the laser spot, owing to the ponderomotive force expelling electrons from the intense region of the laser pulses and exciting the plasma bubble. In addition, the electron beam transverse motion is different from that in the case of the symmetrical laser spot, as shown in Figures 4(h)-4(j), and it no longer oscillates mainly in the $X Y$ plane.

The simulated electron beam profiles are shown in Figures 5(a)-5(d). Figures 5(a)-5(c) are the electron beam profiles at different propagation distances, corresponding to Figures 4(h)-4(j), respectively. These electron beam profile shapes are similar to the cross-section shapes of the plasma bubble, and the three typical simulated electron beam profiles also agree well with the experimental results. In addition, according to the simulation, the laser propagation distance at which the electron beam profile changes from one shape to another is about $200 \mu \mathrm{m}$, as shown in Figures 4(h)-(j). In other words, the electron beam profiles appear as three different kinds of shape, depending on injection positions or acceleration distances. Actually, in our experiment, the processes of injection and acceleration of the electron beam were unstable owing to the fluctuation of laser parameters (energy, duration, contrast, and others) and target parameters (gas density distribution). However, in the case of symmetrical focus, the shape of the electron beam in the experiment and in the simulation was always an ellipse, with the long axis in the laser polarization direction, as shown in Figure 5(d).

To compare the influence of the asymmetrical plasma wakefield on the transverse motion of the accelerated electron beam in the plasma bubble, the phase spaces of $\mathrm{P}_{y}-z$ and
$\mathrm{P}_{z}-y$ corresponding to the electrons of Figures 5(c) and 5(d) are shown in Figures 5(e) and 5(f) and Figures 5(g) and 5(h), respectively. Electrons in the symmetrical plasma wakefield have more momentum in the $y$-direction than in the $z$ direction, as shown in Figures 5(g) and 5(h), resulting in the shape of the electron beam tending to be an ellipse, as shown in Figure 5(d). However, for the asymmetrical plasma wakefield, the maximum momentum in the $y$-direction is approximately equal to that in the $z$-direction, as shown in Figures 5(e) and 5(f), and a majority of electrons have momentum in the $z$-direction larger than that in the case of the symmetrical plasma wakefield, as show in Figures 5(e) and $5(\mathrm{~g})$. Therefore, a majority of electrons have experienced a strong force in the $z$-direction in the asymmetrical transversal wakefield.

\section{Discussion}

To discuss the transverse motion of the accelerated electron beam in the plasma bubble, the transverse forces experienced by the electrons on a cross-section are analyzed. Figures 6(a) and 6(d) show the cross-section of the plasma bubble corresponding to the cases of Figures 5(d) and 5(a), respectively, and Figures 6(b) and 6(e) and Figures 6(c) and 6(f) show the corresponding force distribution of $f_{y} /|q|$ and $f_{z} /|q|$ expressed as

$$
\begin{aligned}
& f_{y}(y, z) /|q|=-E_{y}(y, z)-v_{x} \cdot B_{z}(y, z), \\
& f_{z}(y, z) /|q|=-E_{z}(y, z)+v_{x} \cdot B_{y}(y, z),
\end{aligned}
$$

where $E_{y}$ and $B_{z}$ are the self-generated fields in the plasma bubble and $v_{x}$ is the speed of an electron in the direction of
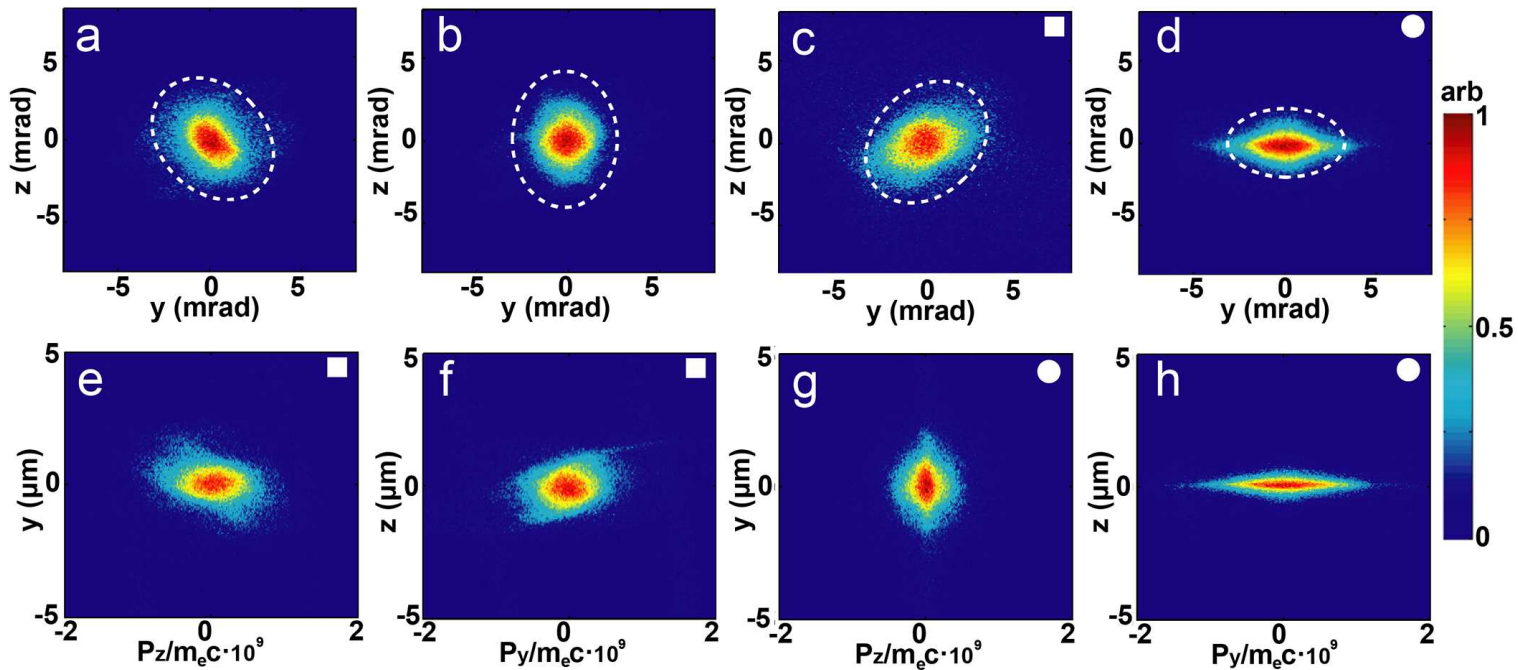

Figure 5. Electron beam spots in 3D-PIC simulations. (a)-(c) Electron beam spots driven by a $45^{\circ}$ slanted elliptical laser focus at different laser propagation distances $(\sim 485 \mu \mathrm{m}, 685 \mu \mathrm{m}$ and $885 \mu \mathrm{m})$ in nitrogen. (d) Corresponding to the case of the circular laser spot at a distance of $\sim 885 \mu \mathrm{m}$. (e), (f) The phase spaces of the $\mathrm{P}_{y}-z$ and $\mathrm{P}_{z}-y$ distributions, respectively, corresponding to the electrons in Figure 4(c). (g), (h) The phase spaces corresponding to the case of Figure 4(d). 
laser propagation. For the symmetrical laser spot, the transverse plasma wakefield is also geometrically symmetrical, as shown in Figures 6(b) and 6(c). The force in the $z$-direction experienced by the electron beam is almost equal to zero in the polarized plane of the maximum laser intensity, as shown in Figure 6(c). Generally, ionization injected electrons will have residual momentum in the electric-field direction of laser pulses. Thus, the injected electrons mainly oscillate in the $y$-direction, driven by the force of $f_{y}$, and the transverse field gradient can reach $100 \mathrm{GV} / \mathrm{m}$, as shown in Figure 6(b). Meanwhile, the slanted elliptical laser spot will destroy the symmetries of the transverse wakefield, as shown in Figures 6(e) and 6(f). Although the ionization injected electron beam initially oscillates in the plane of laser polarization, it will gradually deviate from this direction, as shown in
Figure 6(d). This is because the electron beam experiences a strong force of $f_{z}$ and the field gradient can be up to $50 \mathrm{GV} / \mathrm{m}$. Moreover, the direction of the resultant force tends to be along the long axis of the cross-section of the bubble, resulting in an electron beam shape that is similar to that of the cross-section of the bubble. Also, owing to the laser pulses self-focusing in the plasma channel, the direction of the resultant force will also change with the evolution of the laser intensity distribution and the cross-section of the wakefield.

To compare the motion of the electron beam for the two cases, we tracked 20 electrons individually,and their trajectories are shown in Figures 7(a) and 7(b). In the case of the symmetrical laser spot, these electrons mainly oscillate in the $X Y$ plane with maximum amplitude of $\sim 5 \mu \mathrm{m}$, with
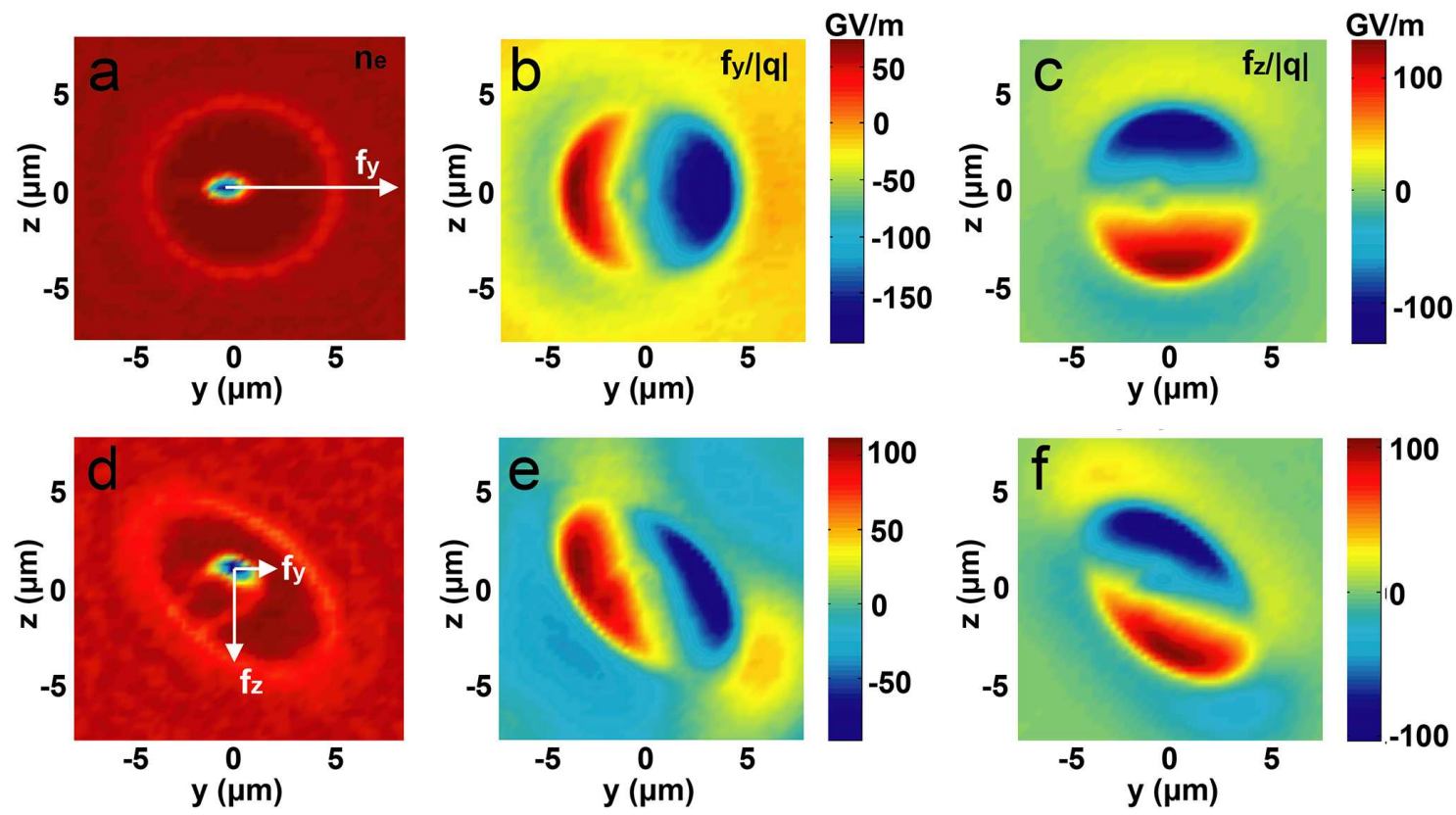

Figure 6. Analysis of transverse force for the electrons in the plasma wakefield. (a), (d) The cross-sections of the plasma bubble corresponding to the circular spot and the elliptical spot, respectively. (b), (c) The transverse force in the directions of $y$ and $z$, respectively, corresponding to (a). (e), (f) The transverse force in the directions of $y$ and $z$, respectively, corresponding to (d).
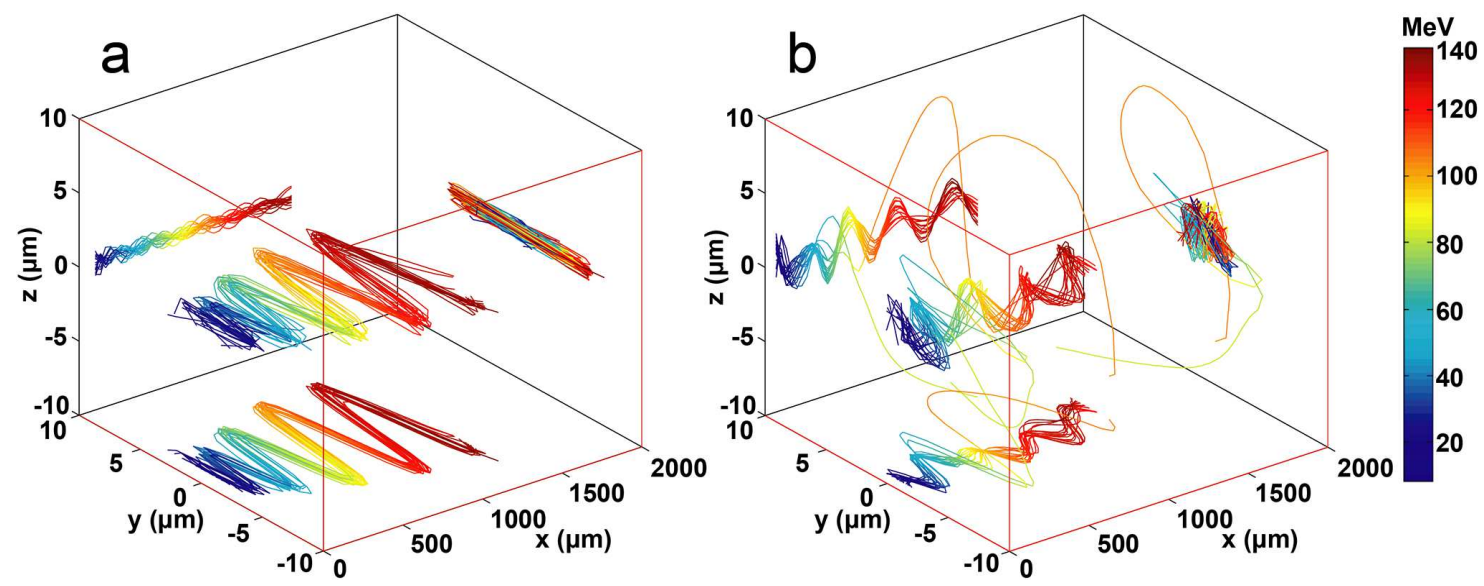

Figure 7. Trajectories of the electrons driven by (a) circular laser focus and (b) elliptical laser focus. 
the amplitude in the $X Z$ plane about $0.5 \mu \mathrm{m}$. However, in the case of the asymmetrical laser spot, these electrons display helical motion, and their oscillation amplitudes are about $3 \mu \mathrm{m}$ in both the $X Y$ and $X Z$ planes. This kind of rotary motion favors the generation of synchrotron radiation from the orbit angular momentum ${ }^{[19,24]}$.

\section{Conclusion}

In conclusion, a simple method of controlling the transverse motion of an electron beam in a plasma bubble is presented. Laser pulses with a power of $100 \mathrm{TW}$ drive a plasma wakefield and accelerate an electron beam in the regime of ionization injection. The transverse motion of the accelerated electron beam can be controlled by changing the intensity distribution of the laser focus by adjusting the posture of the OAP mirror. When the shape of the laser focus changes from circular to slanted elliptical, the geometrical symmetry of the transverse force in the plasma bubble is changed, resulting in the motion of the electron beam changing from undulating to helical. Moreover, the profile of the electron beam also changes with the laser focal spot's shape. The experimental results were verified by 3D-PIC simulations.

This method is expected to conveniently control the transverse motion of an electron beam in a plasma wakefield and to generate synchrotron radiation with orbit angular momentum.

\section{Acknowledgements}

This work was supported by the Science Challenge Project (No. TZ2018005), the National Key R\&D Program of China (No. 2017YFA0403301), the National Natural Science Foundation of China (Nos. 11991073, 11721404, 11805266, 11905289, and 61975229), the Chinese Postdoctoral Science Foundation (No. Y9BK014L51), and the Key Program of CAS (No. XDB17030500).

\section{References}

1. T. Tajima and J. M. Dawson, Phys. Rev. Lett. 43, 267 (1979).

2. J. Osterhoff, A. Popp, Z. Major, B. Marx, T. P. Rowlands-Rees, M. Fuchs, M. Geissler, R. Horlein, B. Hidding, S. Becker, E. A. Peralta, U. Schramm, F. Gruner, D. Habs, F. Krausz, S. M. Hooker, and S. Karsch, Phys. Rev. Lett. 101, 085002 (2008).

3. R. Weingartner, S. Raith, A. Popp, S. Chou, J. Wenz, K. Khrennikov, M. Heigoldt, A. R. Maier, N. Kajumba, M. Fuchs, B. Zeitler, F. Krausz, S. Karsch, and F. Grüner, Phys. Rev. Spec. Top. Accel. Beams 15, 111302 (2012).

4. A. J. Gonsalves, K. Nakamura, C. Lin, D. Panasenko, S. Shiraishi, T. Sokollik, C. Benedetti, C. B. Schroeder, C. G. R. Geddes, J. van Tilborg, J. Osterhoff, E. Esarey, C. Toth, and W. P. Leemans, Nat. Phys. 7, 862 (2011).

5. A. Buck, J. Wenz, J. Xu, K. Khrennikov, K. Schmid, M. Heigoldt, J. M. Mikhailova, M. Geissler, B. Shen, F. Krausz, S. Karsch, and L. Veisz, Phys. Rev. Lett. 110, 185006 (2013).
6. W. Lu, M. Tzoufras, C. Joshi, F. S. Tsung, W. B. Mori, J. Vieira, R. A. Fonseca, and L. O. Silva, Phys. Rev. Spec. Top. Accel. Beams 10, 061301 (2007).

7. E. Esarey, B. A. Shadwick, P. Catravas, and W. P. Leemans, Phys. Rev. E 65, 056505 (2002).

8. D. Umstadter, J. K. Kim, and E. Dodd, Phys. Rev. Lett. 76 , 2073 (1996).

9. J. Faure, C. Rechatin, A. Norlin, A. Lifschitz, Y. Glinec, and V. Malka, Nature 444, 737 (2006).

10. H. Suk, N. Barov, J. B. Rosenzweig, and E. Esarey, Phys. Rev. Lett. 86, 1011 (2001).

11. M. Schnell, A. Savert, I. Uschmann, M. Reuter, M. Nicolai, T. Kampfer, B. Landgraf, O. Jackel, O. Jansen, A. Pukhov, M. C. Kaluza, and C. Spielmann, Nat. Commun. 4, 2421 (2013).

12. A. Popp, J. Vieira, J. Osterhoff, Z. Major, R. Horlein, M. Fuchs, R. Weingartner, T. P. Rowlands-Rees, M. Marti, R. A Fonseca, S. F. Martins, L. O. Silva, S. M. Hooker, F. Krausz, F. Gruner, and S. Karsch, Phys. Rev. Lett. 105, 215001 (2010).

13. S. Corde, C. Thaury, A. Lifschitz, G. Lambert, K. Ta Phuoc, X. Davoine, R. Lehe, D. Douillet, A. Rousse, and V. Malka, Nat. Commun. 4, 1501 (2013).

14. C. McGuffey, A. G. Thomas, W. Schumaker, T. Matsuoka, V. Chvykov, F. J. Dollar, G. Kalintchenko, V. Yanovsky, A. Maksimchuk, K. Krushelnick, V. Y. Bychenkov, I. V. Glazyrin, and A. V. Karpeev, Phys. Rev. Lett. 104, 025004 (2010).

15. A. Pak, K. A. Marsh, S. F. Martins, W. Lu, W. B. Mori, and C. Joshi, Phys. Rev. Lett. 104, 025003 (2010).

16. E. Oz, S. Deng, T. Katsouleas, P. Muggli, C. D. Barnes, I. Blumenfeld, F. J. Decker, P. Emma, M. J. Hogan, R. Ischebeck, R. H. Iverson, N. Kirby, P. Krejcik, C. O'Connell, R. H. Siemann, D. Walz, D. Auerbach, C. E. Clayton, C. Huang, D. K. Johnson, C. Joshi, W. Lu, K. A. Marsh, W. B. Mori, and M. Zhou, Phys. Rev. Lett. 98, 084801 (2007).

17. M. Chen, E. Esarey, C. B. Schroeder, C. G. R. Geddes, and W. P. Leemans, Phys. Plasmas 19, 033101 (2012).

18. J. Wang, J. Feng, C. Zhu, Y. Li, Y. He, D. Li, J. Tan, J. Ma, and L. Chen, Plasma Phys. Control. Fusion 60, 034004 (2018).

19. J. Feng, Y. Li, X. Geng, D. Li, J. Wang, M. Mirzaie, and L. Chen, Plasma Phys. Control. Fusion 62, 105021 (2020).

20. A. Döpp, B. Mahieu, A. Lifschitz, C. Thaury, A. Doche, E. Guillaume, G. Grittani, O. Lundh, M. Hansson, J. Gautier, M. Kozlova, J. P. Goddet, P. Rousseau, A. Tafzi, V. Malka, A. Rousse, S. Corde, and K. Ta Phuoc, Light Sci. Appl. 6, e17086 (2017).

21. K. Huang, D. Z. Li, W. C. Yan, M. H. Li, M. Z. Tao, Z. Y. Chen, X. L. Ge, F. Liu, Y. Ma, J. R. Zhao, N. M. Hafz, J. Zhang, and L. M. Chen, Appl. Phys. Lett. 105, 204101 (2014).

22. E. Esarey, C. B. Schroeder, and W. P. Leemans, Rev. Mod. Phys. 81, 1229 (2009).

23. J. Luo, M. Chen, M. Zeng, J. Vieira, L. L. Yu, S. M. Weng, L. O. Silva, D. A. Jaroszynski, Z. M. Sheng, and J. Zhang, Sci. Rep. 6, 29101 (2016).

24. C. Thaury, E. Guillaume, S. Corde, R. Lehe, M. Le Bouteiller, K. Ta Phuoc, X. Davoine, J. M. Rax, A. Rousse, and V. Malka, Phys. Rev. Lett. 111, 135002 (2013).

25. H. X. Chang, B. Qiao, T. W. Huang, Z. Xu, C. T. Zhou, Y. Q. Gu, X. Q. Yan, M. Zepf, and X. T. He, Sci. Rep. 7, 45031 (2017).

26. K. Huang, Y. F. Li, D. Z. Li, L. M. Chen, M. Z. Tao, Y. Ma, J. R. Zhao, M. H. Li, M. Chen, M. Mirzaie, N. Hafz, T. Sokollik, Z. M. Sheng, and J. Zhang, Sci. Rep. 6, 27633 (2016).

27. W. Yan, L. Chen, D. Li, L. Zhang, N. A. Hafz, J. Dunn, Y. Ma, K. Huang, L. Su, M. Chen, Z. Sheng, and J. Zhang, Proc. Natl. Acad. Sci. USA 111, 5825 (2014).

28. T. Hosokai, K. Kinoshita, T. Watanabe, K. Yoshii, T. Ueda, A. Zhidkov, M. Uesaka, H. Kotaki, M. Kando, and K. Nakajima, 
in Advanced Accelerator Concepts (American Institute of Physics, Melville, 2002), pp. 628-633.

29. A. E. Hussein, J. Ludwig, K. Behm, Y. Horovitz, P. E. Masson-Laborde, V. Chvykov, A. Maksimchuk, T. Matsuoka, C. McGuffey, V. Yanovsky, W. Rozmus, and K. Krushelnick, New J. Phys. 20, 073039 (2018).
30. G. J. Williams, B. R. Maddox, H. Chen, S. Kojima, and M. Millecchia, Rev. Sci. Instrum. 85, 833 (2014).

31. M. Chen, Z. Sheng, J. Zheng, Y. Ma, and J. Zhang, Chin. J. Comput. Phys. 25, 43 (2008).

32. W. M. Wang, P. Gibbon, Z. M. Sheng, and Y. T. Li, Phys. Rev. E 91, 013101 (2015). 